\title{
Interaction of stimulus and contextual information during reading: Identifying words within sentences
}

\author{
THOMAS SANOCKI, KAREN GOLDMAN, JENNIFER WALTZ, CAMERON COOK, \\ WILLIAM EPSTEIN, and GREGG C. ODEN \\ University of Wisconsin, Madison, Wisconsin
}

\begin{abstract}
In general, studies on the effects of a sentence context on word identification have focused on how context affects the efficiency of processing a single target word, presented separately from the context. Such studies probably would be incapable of measuring contextual facilitation resulting from cascaded or parallel processing of neighboring words within a sentence. To measure these and other types of facilitation, we presented entire phrases and sentences for subjects to read as fast as possible and to monitor for nonwords. Subjects read at rates representative of natural reading. Experiment 1 demonstrated a large contextual facilitation effect on decision time. Experiment 2 showed that facilitation is caused by specific semantic information and, perhaps to a greater degree, by nonpredictive syntactic information. Experiment 3 showed that the amount of facilitation is greater than could be accounted for by separate contributions from autonomous word level and sentence level processes. These results present difficulties for an autonomous model of reading, but are consistent with interactive models, in which the results of ongoing sentential analyses are combined with stimulus information to identify words.
\end{abstract}

An important issue in the study of reading is how the meaning of the context affects particular aspects of the processing of individual words. For example, it is possible that the sensory analysis of a word could be affected by context, but little evidence supports this notion (for counterevidence, see, e.g., Schvaneveldt \& McDonald, 1981, and Zola, 1984), and most currently popular models of reading assume that the sensory analysis is independent of the context. Similarly, research on lexical ambiguity indicates that both contextually appropriate and inappropriate meanings of an ambiguous word become activated (e.g., Oden \& Spira, 1983; Seidenberg, Tanenhaus, Leiman, \& Bienkowski, 1982; Swinney, 1979), and therefore many models assume that context has little or no effect on the initial activation of words and their meanings by featural information (e.g., Forster, 1979; MarslenWilson \& Welsh, 1978; Oden \& Spira, 1983).

The activation of multiple senses of ambiguous words is consistent with models in which the entire word identification process is autonomous and self-contained, with only featural information being used to identify the stimulus as a particular word (e.g., Forster, 1979; Seidenberg et al., 1982; Stanovich \& West, 1983b). According to

This research was supported by NSF Grant BNS80-14316 to Gregg Oden. We thank Art Glenberg, Jola Jakimak, Jay Rueckl, and Ed Shoben for helpful comments on this research, and Lola Lopes for the use of her laboratory. Request for reprints can be sent to the first or last author at the Department of Psychology, University of Wisconsin, Madison, WI 53706 . these models, the only context effect on word identification during normal reading is a more limited, word level effect - priming between highly related words (e.g., Forster, 1979; Seidenberg et al., 1982; Stanovich \& West, $1983 \mathrm{~b}$ ). This effect (hereafter referred to as word priming) is to be distinguished from more general context effects that involve analyses of a sentence's meaning.

An alternative interpretation of these lexical ambiguity studies is provided by interactive models of reading (e.g., Oden \& Spira, 1983). In these models, the initial featural analysis of a word causes a "cohort" of units representing words and their meanings to become activated. These words are then considered in parallel during a word identification process that involves both stimulus and contextual information (e.g., Marslen-Wilson \& Welsh, 1978; Oden, 1984). In these models, contextual information is not used in the initial cohort activation process, but it is used subsequently in resolving a word's identity. Therefore, it is important to distinguish between the initial activation of meanings and the eventual resolution of a word's identity and meaning (see also Oden \& Spira, 1983).

The focus of this paper is on the resolution of words' identities during reading. Our main objective was to test autonomous (e.g., Forster, 1979) and interactive (e.g., Marslen-Wilson \& Welsh, 1978; Oden, 1984; Rumelhart \& McClelland, 1982) models of this process. However, before testing models, it is important to develop an appropriate methodology.

The most commonly used paradigm for examining the 
possibility of context effects on word resolution has been the sentence priming paradigm, in which a subject is presented with either a sentence context or a noninformative baseline context, followed by a single target word that can complete the sentence context (e.g., Stanovich \& West, 1983b; Tulving \& Gold, 1963). The speed or accuracy of a response to the target word is measured, and it is assumed that if context facilitates word identification, then the response will be quicker or more accurate with targets that follow appropriate sentence contexts than with words that follow baseline contexts.

Artifactual influences and more ecologically representative variables affect the amount of facilitation obtained with this task (see e.g., Fischler \& Bloom, 1979, Forster, 1981, Sanocki \& Oden, 1984, and Stanovich \& West, 1983b). One potential problem is that facilitation effects may occur because word identification is unnaturally slowed, allowing slow-acting contextual processes to come into play (see, e.g., Mitchell, 1982, and Stanovich \& West, 1983b). Previous studies are also limited because most of them have focused on a limited class of target words, namely, words that occur in the final position of sentences. Context effects may be smaller or nonexistent for other types of words (see Stanovich \& West, 1983a). Finally, most studies have used uppercase targets, eliminating letter shape as a type of stimulus information and thereby increasing subjects' reliance on context (see Stanovich \& West, 1983b, and Underwood \& Bargh, 1982).

One potentially important limitation of sentence priming studies has not been discussed previously in this literature. When the processing time for a single target word is measured, the word must be processed separately from its context. This would preclude facilitation that might result from a word being processed at the same time as other words in the immediate context. For example, the processing of a particular word $\mathrm{n}$ could begin as the reader picks up information about it parafoveally, while fixating words to its left. This reduces the number of features that must be picked up when word $n$ is fixated, decreasing the duration of the fixation and allowing the reader to fixate word $n+1$ relatively soon. The reader may fixate word $n+1$ while continuing to process word $n$ at levels that follow feature extraction. This overlapping, cascaded processing means that more than one word is processed at the same time. It is also possible that information about two adjacent words is sometimes gathered during the same fixation. These two words could be processed in parallel.

Evidence supports these speculations. Contextual information reduces the duration of eye fixations (S. F. Ehrlich \& Rayner, 1981; Zola, 1984), and can interact with parafoveal information to cause facilitation (McClelland \& O'Regan, 1981; see also Balota \& Rayner, 1983). The idea of overlapping, cascaded processing has considerable theoretical and empirical support (McClelland, 1979), and support in reading studies (K. Ehrlich \& Rayner, 1983). The parallel processing of adjacent words is implicated by findings of "backward" priming (Kiger \& Glass, 1983) and letter migration (Mozer, 1983). Finally, since readers often do not fixate short function words (Rayner, 1983), readers may gather information about them while fixating other words.

In sum, the identification of the set of words a sentence comprises may be greatly speeded because they can be processed in a cascaded and sometimes parallel manner. However, the total amount of time during which a single word within the sentence is processed may not be reduced at all (in fact, it may be increased). Facilitation arising from cascaded or parallel processing would be very difficult to measure with paradigms in which target words are presented individually, separately from their context. This problem could be serious because investigators have used results from sentence priming studies to argue that the meaning of the context does not facilitate word identification during reading (e.g., Fischler \& Bloom, 1979; Forster, 1979, 1981; Stanovich \& West, 1983a, 1983b).

The first goal in the present experiments was to develop a new task for investigating context effects during reading. The task should (1) allow subjects to process words in a cascaded or parallel manner, and yet (2) require them to process each word for meaning. We would also want to avoid the other methodological problems referred to earlier. In particular, we wanted to induce subjects to read at rates typical of natural reading, so that contextual influences are not triggered by an unnatural slowing of the word identification process (Mitchell, 1982; Stanovich \& West, 1983b). In our task, entire sentences and word lists were presented for subjects to read and to monitor for nonwords. The dependent measure was "scanning time," the amount of time needed to decide whether or not the string consisted solely of words. Experiment 1 demonstrates a basic contextual facilitation effect, and Experiment 2 investigates the contributions to facilitation of general syntactic information and specific semantic information. After these two experiments, we will consider how the results can be handled by "autonomous" models of reading (e.g., Forster, 1979) and "interactive" models (e.g., Marslen-Wilson \& Welsh, 1978; Oden, 1984; Rumelhart \& McClelland, 1982). Then the models will be tested directly in Experiment 3.

\section{EXPERIMENT 1}

The purpose of the first experiment was to see whether a context effect could be obtained with the scanning task. The strings were either sentences or scrambled versions of the same sentences (see Table 1). In this experiment, $80 \%$ of the strings consisted entirely of words ("legal strings"'), and the remaining strings included a single nonword. If a meaningful context facilitates word processing, then the strings of words should be read more quickly when they form sentences than when they are in a scrambled order.

\section{Method}

Materials. The critical stimuli were 48 pairs of legal strings, ranging in length from 5 to 10 words and averaging 6.25 words. The strings within a pair contained the same words, but one member 
Table 1

Representative Stimuli from Experiment 1

Critical pairs (legal strings)
1. Ann bought her neighbor a book. (sentence)
Bought neighbor a her book Ann. (scrambled version)
2. Tom sees the old bus.
Old the sees Tom bus.
3. The lamp was set on the floor.
On was lamp the floor set the.
Nonword items (distractor strings)
1. Many residents hate the cold lontards. (sentence)
2. The by nured sun was Mary. (scrambled)

formed a sentence, whereas the other member had the words in a scrambled order. All sentences had a single clause but otherwise varied in surface structure. Strong preexperimental associations and semantic relations between content words within a sentence were avoided. A subject saw only one member from each pair of "yes" strings. Examples of the strings are shown in Table 1.

Six sentences and six scrambled strings were constructed to be used as distractors. In each of these, one word was replaced with a pronounceable, orthographically legal nonword of approximately the same length (see Table 1). The ordinal position of the nonword within the string was varied. Additional strings were also constructed for use as practice stimuli.

Procedure and Apparatus. The subjects were tested individually in sessions lasting about $25 \mathrm{~min}$. The type of string was blocked, with the block-type order counterbalanced across subjects. The first two blocks were practice, as were the first five trials within each of the four test blocks. A test block included 12 critical legal trials and 3 distractor trials, with the strings appearing in a randomized order.

The subjects were seated approximately $80 \mathrm{~cm}$ from a 9-in. video monitor driven by an Apple II microcomputer. At the start of a trial, a string appeared on the screen, printed with appropriate punctuation, in upper- and lowercase letters formed on a $7 \times 8$ dot matrix. The subjects were told to read the string as quickly as possible, and to press the "yes" key if it contained all words or the "no" key if it contained a nonword. The subjects used their dominant hands to press the "yes" key. Feedback was given after erroneous responses.

Subjects. Twenty-two University of Wisconsin undergraduates, 11 in each counterbalance group, served as subjects for extra course credit.

\section{Results}

Of main interest was a comparison based on the subjects' mean scanning times for legal strings in the two context conditions. ${ }^{1,2}$ Sentence strings were scanned an average of $310 \mathrm{msec}$ faster than scrambled strings $[F(1,21)$ $=27.34, p<.001]$. This suggests that a meaningful sentence context does facilitate the identification of words. The average amount of facilitation per word in the sentences was $50 \mathrm{msec}$, although the amount probably varied considerably from word to word; for example, the facilitation effect may have been greater for content words or less for words near the beginning of the sentence.

The scanning times and error rates in each condition are shown in Table 2 . In a $2 \times 2$ analysis of variance on scanning times, the main effect of context was reliable $[F(1,21)=14.60, p<.001]$ and did not change with the legality of the string (i.e., the interaction was nonsignificant $[F(1,21)=2.44, p>.10]$. For the error data, neither the context main effect $[F(1,21)=1.15, p>$ .25 ] nor the interaction $(\mathrm{F}<1)$ was reliable.

Since the sentences averaged 6.25 words in length, the scanning rate for sentences was 235 words per minute. Because this is well within the range of normal reading rates, it provides evidence that the present task was fairly natural. If the task had posed unnatural demands on readers, it seems that the reading rate would have been retarded relative to normal reading. In addition, because scanning is rapid, it is less likely that the subjects would have had time to employ task-specific strategies for using context.

\section{EXPERIMENT 2}

A sentence contains many types of information, and it is not clear which of these types of information contributed to the large context effect obtained in Experiment 1. We can make at least a rough distinction between (1) lowconstraint syntactic or semantic information, with which large classes of words are compatible, and (2) above and beyond this, more predictive semantic information, with which only a few words may be highly compatible. The purpose of Experiment 2 was to test whether or not each of these types of information causes facilitation.

A facilitatory effect of low-constraint contextual information would be of particular theoretical interest, because it would implicate a linguistically powerful mechanism. For example, a contextual phrase such as "He really needed to put some" could be completed by phrases that consist of different words, having very different meanings. A facilitatory effect of such a context would implicate a high-level mechanism that could affect more words than word level mechanisms (e.g., Becker, 1980; Neely, 1977) could affect. Evidence of such facilitatory effects in sentence priming studies is mixed; facilitatory effects have been found in some studies (McClelland \& O'Regan, 1981; Sanocki \& Oden, 1984; Schwanenflugel \& Shoben, in press) but not others (Fischler \& Bloom, 1979; Forster, 1981).

At the same time, it would be of interest to test whether or not predictive semantic information has effects above and beyond the effects of low-constraint syntactic information. Highly constraining information has been shown to cause facilitation (e.g., Fischler \& Bloom, 1979; Tulving \& Gold, 1963), but it is not clear that such effects occur during normal reading (for arguments to the contrary, see Fischler \& Bloom, 1979, Forster, 1981, Mitchell, 1982, and Mitchell \& Green, 1978).

Table 2

Mean Scanning Time (in Milliseconds) and Percent Errors (PEs) in Experiment 1

\begin{tabular}{lcccr} 
& Sentences & PE & Scrambled Strings & PE \\
\hline Legal Items & 1596 & 1.0 & 1906 & 1.5 \\
Distractor Items & 1981 & 16.7 & 2166 & 20.4 \\
\hline
\end{tabular}


Table 3

Representative Legal Sentence Sets from Experiment 2

Syntactic comparison
1. While walking late at night he (sentence context)
saw a bright full moon. (target phrase)
heard some drunks walking home. (target phrase)
He really needed to put some
gas in his car.
new locks on the doors.
The trip through France
included stops at many famous museums.
was a great chance to drink wine and party.
3.
Semantic comparison
The Grand Canyon is (predictive context)
He thought that the back yard was (plausible context)
a very beautiful sight. (target phrase)
The doctor
The man
made a mistake during the operation.
The cow was
The company was
producing less milk than usual.

To address these issues in Experiment 2, contextual information was manipulated via a sentence priming procedure. Subjects first read a prime string (the first few words of a sentence, e.g., "The boy from next door") and then scanned a target string that completed the sentence (e.g., "was hurrying to the fire"). This procedure introduces an unnatural interval between the processing of the context and the target string, but it has the advantage of permitting powerful and yet precise control over contextual information.

\section{Method}

Design and Materials. The syntactic comparison concerned the effects of low-constraint syntactic or semantic information. The contrast was between scanning times when the target strings were preceded by nonpredictive sentential contexts (syntactic context) and when they were preceded by a baseline context (" $x x x x x x$ $\operatorname{xxxxxx} x \times x x x x$ be ready"). To ensure that any facilitation effects would not be due to the predictability of the target string, we used two different target strings with each context, neither of which was highly predictable. The two target strings had different meanings, so that even if one target did happen to be predictable from the context, the other could not also be predictable. Examples are shown in Table 3. Twenty critical sentence sets were created. A given subject saw both targets in a sentence set, one preceded by the syntactic context and one preceded by the baseline context; the pairings were counterbalanced across subjects. The targets for the syntactic comparison averaged 5.4 words in length.

The semantic comparison concerned the effects of more predictive semantic information. The contrast was between the effects of two types of contexts, both of which were congruous with the target. Each sentence set consisted of (1) a target string, (2) a "predictive" context, which had strong implications about the meaning of the target, and (3) a "plausible" context, which had weak implications about the meaning of the target. Examples are shown in Table 3 . For this comparison, 40 critical sentence sets were created. The predictiveness of the contexts was confirmed in a separate pilot study ${ }^{3}$ In the main study, a subject saw every target but only one of the two contexts in a sentence set; the pairings were counterbalanced. The targets averaged 6.1 words in length.
For each of the four conditions described above, five distractor contexts and strings were constructed. In each target, one word was replaced with a pronounceable, orthographically legal nonword that resembled the original word in shape and length. In addition, 20 practice sets of contexts and targets were used, as well as 10 nonsense "catch" contexts for use in a secondary task designed to encourage reading of the context (see below).

Procedure. The subjects were tested individually with the same apparatus as in Experiment 1, in sessions lasting about $30 \mathrm{~min}$. The materials for the two comparisons appeared in an intermixed, randomized order. At the beginning of each trial, a context phrase appeared on the screen. In addition to the test and practice contexts, 10 contexts that did not make sense appeared during the experiment. The subjects were told that some "catch" contexts were included in order to test whether or not they were reading carefully. The subjects were to press the "yes" key if the context did make sense or the "no/catch" key otherwise. If the context was sensible and the subject pressed the "yes" key, then the context was erased and, between 900 and $1,400 \mathrm{msec}$ later, the target appeared on the screen.

Subjects. Twenty-four University of Wisconsin-Madison undergraduates, 12 in each counterbalance group, served as subjects for extra course credit.

\section{Results}

Syntactic comparison. The syntactic comparison concerns whether or not low-constraint syntactic information can facilitate the processing of words. There was indeed a facilitatory effect of syntactic information, because the target phrases were scanned an average of $180 \mathrm{msec}$ faster when they were preceded by syntactic contexts than when they were preceded by baseline contexts $[F(1,23)=$ $30.25, \mathrm{p}<.001]$. The strings following syntactic contexts were scanned at a rate of 232 words per minute; the facilitatory effect averaged $33 \mathrm{msec}$ per word.

As noted above, two target phrases were used with each syntactic context, in order to ensure that the targets were not semantically predictable. It is logically possible that half of the targets were semantically predictable, and that the obtained effect was due to a mixture of predictable items with large facilitatory effects and unpredictable items with small or negative effects. However, the facilitatory effect was consistent across items, obtaining for 33 of the 40 targets.

The mean scanning times and error rates for all conditions are shown in Table 4. For scanning time, the main effect of syntactic context was reliable $[F(1,23)=42.85$, $\mathrm{p}<.001]$ and consistent across the legality factor $(\mathrm{F}<$ 1 for the interaction). For errors, there was no main ef-

Table 4

Mean Scanning Time (in Milliseconds) and Percent Errors (PEs) in Experiment 2

\begin{tabular}{|c|c|c|c|c|}
\hline & \multicolumn{2}{|c|}{ Legal Strings } & \multicolumn{2}{|c|}{ Distractor Strings } \\
\hline & Mean & $\mathrm{PE}$ & Mean & PE \\
\hline \multicolumn{5}{|c|}{ Syntactic Comparison } \\
\hline Sentence Context & 1402 & 0.8 & 1390 & 6.7 \\
\hline Baseline Context & 1582 & 2.9 & 1604 & 2.5 \\
\hline \multicolumn{5}{|c|}{ Semantic Comparison } \\
\hline $\begin{array}{l}\text { Predictive context } \\
\text { Plausible Context }\end{array}$ & $\begin{array}{l}1507 \\
1603\end{array}$ & $\begin{array}{l}1.2 \\
1.0\end{array}$ & $\begin{array}{l}1447 \\
1385\end{array}$ & $\begin{array}{l}18.3 \\
14.2\end{array}$ \\
\hline
\end{tabular}


fect of context $(F<1)$, but the interaction with legality was reliable $[F(1,23)=7.28, p<.05]$. The interaction stems from the fact that, for legal strings, syntactic context increased accuracy $[\mathrm{F}(1,23)=5.37, \mathrm{p}<.05]$, whereas, for distractor strings, the effect was in the opposite direction [but unreliable; $F(1,23)=1.72, p>.10$ ]

Semantic comparison. The semantic comparison concerns whether or not contexts that have strong implications about the meaning of the target cause facilitation beyond that caused by plausible contexts. Indeed, the target phrases were scanned $96 \mathrm{msec}$ faster when they were preceded by predictive contexts than when they were preceded by plausible contexts $[\mathrm{F}(1,23)=15.8, \mathrm{p}<$ $.001]$. The reading rates were 243 and 228 words per minute, respectively, and the semantic effect averaged $16 \mathrm{msec}$ per word. The fact that this effect was obtained at the same time as the syntactic effect indicates that the semantic effect did not result from a task-specific strategy for using predictive information. ${ }^{4}$

The means and error rates for each condition are shown in Table 4. For scanning time, the main effect of context was not reliable $(\mathrm{F}<1)$, but the interaction with legality was $[F(1,23)=5.39, p<.05]$. Legal strings were scanned faster when preceded by predictive contexts (as reported above), but for distractor strings the effect was unreliable $(F<1)$ and in the opposite direction. For error rates, neither the main effect of context $(F<1)$ nor the interaction $[F(1,23)=2.88, p>.10]$ was reliable.

\section{Discussion of Experiments 1 and 2}

The scanning task is sensitive to contextual facilitation effects. The robust facilitation effects suggest that it may be necessary to reconsider conclusions from sentence priming studies in which the meaning of the context has not facilitated word identification (e.g., Fischler \& Bloom, 1979; Forster, 1981; Stanovich \& West, 1983b). However, before doing so, it is necessary to consider the meaning of the effects within particular psychological models.

In the interactive model, the word identification process involves several distinct subprocesses (see, e.g., MarslenWilson \& Welsh, 1978, Oden, 1984, and Rumelhart \& McClelland, 1982). First, stimulus information is extracted from a word, causing the activation of a "cohort" of word candidates (Marslen-Wilson \& Welsh, 1978). Stimulus analyses continue, independently of higher level processing, while a contextual evaluation process analyzes the contextual appropriateness of each cohort member (see Oden, 1983). A cohort word's activation depends on both stimulus analyses and contextual analyses, and is a multiplicative function of the degree to which it is consistent with stimulus information and the degree to which it is appropriate for the context (e.g., Oden \& Spira, 1983; Rueckl \& Oden, 1983). Since the context usually supports a relatively small subset of words, less stimulus information is needed for a decision between members of this subset than if context were not used. Thus, in contrast to topdown models (e.g., Smith, 1971), the interactions in this model are subtle yet general. The stimulus analysis per se is not affected by context; it is simply terminated sooner because the system can "assume" that incomplete stimulus information, when combined with contextual information, would be enough to correctly identify words.

The sentence advantage in Experiment 1 occurs because contextual constraints can be used with sentences but not with scrambled strings. As indicated by the syntactic facilitation effect in Experiment 2, even rather general syntactic constraints facilitate word identification. The semantic effect in Experiment 2 is also consistent with the model, but the effect does not mean that subjects were predicting upcoming words. Rather, it indicates that the words in the target phrases had a greater degree of consistency with the predictive contexts than with the plausible contexts (see Oden, 1984, and Oden \& Spira, 1983). Accordingly, predictive contexts provided stronger support for the words in the target phrases. The fact that the syntactic effect was considerably larger in magnitude (33 msec per word) than the semantic effect (16 msec per word) suggests that the most important function of the contexts was to provide general constraints on the interpretation of the target phrases, rather than to put narrow constraints on their exact meaning.

Alternative explanations stem from the autonomous model of reading (Forster, 1979, 1981; see also Seidenberg et al., 1982, Seidenberg, Waters, Sanders, \& Langer, 1984, and Stanovich \& West, 1983b). In this model, the autonomous word identification process uses stimulus information to produce an object representing a particular word. This object serves as an "address" for higher level autonomous processes, which access the word's class and meaning. As words in a sentence are identified, higher level processes produce a higher level object that represents a sentence. The model also includes a general processor that accepts outputs from a particular autonomous processor and initiates a response based on those outputs.

Theorists advocating the autonomous model have argued that during normal reading, word identification occurs too quickly to be affected by semantic or syntactic analyses (e.g., Forster, 1979; Mitchell, 1982; Seidenberg et al., 1982; Stanovich \& West, 1983b). In fact, Forster $(1979,1981)$ argued that the word identification process is usually not facilitated at all by context, although other theorists have argued that priming within the lexicon is a pervasive phenomonon (e.g., Seidenberg et al., 1982; Stanovich \& West, 1983b).

The word priming explanation centers on the assumption that when words are identified, activation spreads from the representations of their meanings to prime words with related meanings (Collins \& Loftus, 1975). However, it is unlikely that existing models of word priming can explain all of the 50-msec-per-word facilitation effect in Experiment 1, because the sentences were constructed to have few, if any, highly related words (see, e.g., Table 1). In the literature, priming effects have been small (about 30 to $50 \mathrm{msec}$ ) and have been reported only 
for highly related content words (e.g., Seidenberg et al., 1984; Stanovich \& West, 1983a). Stanovich and West (1983a, 1983b), in explaining findings of no facilitation (e.g., Fischler \& Bloom, 1979; Stanovich \& West, 1983a), argued that priming is not likely to occur when the context contains few words highly related to the target word.

In Experiment 2, the subjects could read the contexts at a slow pace. Sentence level processes may become involved when reading is slowed (see Mitchell, 1982, and Stanovich \& West, 1983b), so a second type of priming is possible-priming caused by concepts activated in sentential analyses. However, although the semantic effect can be explained by such priming, the syntactic effect cannot, because it is unlikely that the syntactic contexts could activate enough concepts to prime the words in their target phrases. Each syntactic context had two target phrases, consisting of different words having different meanings. Such a variety of words would not be primed because, as noted above, priming is assumed to occur only between highly semantically related words and concepts (see, e.g., Neely, 1977, Seidenberg et al., 1984, and Stanovich \& West, 1983b).

In sum, priming does not seem to explain the present set of results. An alternative account is given by Forster (1979), in a discussion of some data from an experiment very similar to Experiment 1 here. According to Forster, the entire difference between conditions results from differences in the decision process. Forster noted that when subjects read a sentence in the scanning task, they could draw on the fact that if the items formed a proper sentence, then the string did not contain a nonword. Within his framework, Forster argued that subjects could read the entire string and respond "yes" (legal string) if sentence level processes produced a representation of the sentence, or "no" otherwise. However, with scrambled sentences, such a strategy cannot be used; subjects can use only outputs from the word level and make a word-nonword decision for each item. These decisions are assumed to take a relatively long time. Thus, Forster argued that words are identified equally fast in scrambled strings and sentences, but a sentence advantage arises because subjects make only one (sentence level) decision with a sentence but many (word level) decisions with scrambled strings.

If sentence level decisions were also used in Experiment 2, then the semantic effect can be explained by assuming that sentence level decisions are easier when the contexts predict the meaning of the targets than when they do not. The syntactic effect can be explained by assuming that sentence level decisions are easier with syntactic contexts than with baseline contexts.

In Forster's (1979) model, decisions are made by the general processor, on the basis of complete outputs from a single level. This follows from the idea of autonomous processors that specialize in their own particular type of knowledge. Each autonomous processor is a highly limited, totally dedicated "microprocessor" that is "programmed to perform highly specific tasks in a quite inflexible manner" (Forster, 1979, p. 33). Since the general processor would not have access to the programming (knowledge) of the autonomous processors, it would not have the knowledge necessary for interpreting intermediate results from autonomous processors. And it could not combine the qualitatively different outputs from different processors.

In contrast, the interactive model assumes that partial lexical level and sentence level analyses are combined to identify words during reading. Therefore, it follows from the interactive model that these two sources of information would be combined during decision making in the scanning task. These differing notions about the decision process can be tested by examining the relation between the position of nonwords and scanning time. If subjects scan the entire string and then make a decision on the basis of the output from the sentence level, scanning time should be related to the length of the entire string but not to the position of the nonword. However, if subjects combine lexical and semantic information during the processing of each word, scanning time should be related to the position of the nonword but not to the length of the string. These predictions were tested in Experiment 3, along with a more general prediction about scanning decisions.

\section{EXPERIMENT 3}

As is apparent in the preceding discussion, a fundamental difference between the models is in how different levels of information are combined. In interactive models (e.g., Marslen-Wilson \& Welsh, 1978; Oden, 1984; Rumelhart \& McClelland, 1982), stimulus analyses and contextual analyses are combined to identify words during reading, so these types of information would be combined in the processing of each item during scanning. In contrast, in the autonomous model, each autonomous processor specializes in a particular type of knowledge, accepting inputs from only the immediately lower level and making a complete, specialized output (Forster, 1979). The general processor must consider the qualitatively different outputs from different processors separately. Therefore, as in Forster's explanation described above, word level and sentence level decisions are separate even when both levels are making decisions about the same string.

We can conduct a general test of these models by examining scanning decisions determined by (1) only word level information, (2) only sentence level information, and (3) both levels of information (the both condition). Consider first the autonomous model (Forster, 1979). In the both condition, an autonomous system could use results from either level of analysis, but because each level is separate and autonomous, their results could not be combined. At best, the system simply would respond as soon as a decision was completed at either level. Since autonomous word level or sentence level processes underlie these decisions, we can estimate the speed of this word and sen- 
tence level "race" by pooling data from conditions in which only word level and only sentence level information is available (following Miller, 1981, 1982). An interactive system, in contrast, would combine word and sentence level information, and therefore decisions in the both condition could occur before enough processing had been done for either level to produce a decision on its own. Therefore, the interactive model predicts that decisions in the both condition should be faster than decisions based on a race of autonomous processes.

An additional purpose of Experiment 3 was to examine context effects with more natural sentences, representative of the wide variety of prose styles found in everyday reading. Almost all previous context effect experiments (including Experiments 1 and 2) have involved sentences constructed by experimenters. The generality of the results to more natural texts is, therefore, open to question.

\section{Method}

In three different conditions, subjects were presented with either entire sentences or scrambled versions of the same sentences. In each condition, the task was to read the string of words and search for a particular type of "violation." In contrast to the first two experiments, the subjects were to respond "yes" (violation) as quickly as possible when a violation was found, or "no" upon finishing legal strings, which did not contain a violation. Because of the focus on the detection of violations, $60 \%$ of the strings contained a violation.

Design and Materials. In the sentence condition, the response was determined by sentence level information. The target strings were sentences, and the violations were single words that were $a b$ viously inappropriate for the sentence. Examples of the stimuli are shown in Table 5. In the both condition, the response was determined by both word level and sentence level information. In this condition, the sentences used in the sentence condition served as target strings, but the violations were now nonwords, constructed by replacing one letter in each of the words that served as violations in the sentence condition (see Table 5). These nonwords are a violation at the word level and also at the level of sentence meanings. In the word condition, only word level information was available to the subjects, so the response was determined by word level information. The target strings were scrambled lists, made by scrambling the sentences used in the other two conditions (see Table 5). The violations were the nonwords used in the both condition. Each violation appeared in the same ordinal position as in the other two conditions.

A list of 210 sentences was compiled from four different anthologies of contemporary American short stories. The sentences were chosen by selecting every fifth sentence from every fifth page. Sentences were not used if they were fewer than 5 words or more than 80 characters in length, if they were obscene or contained sexual content, or if their meanings were not apparent independently of the context. Of the 210 sentences selected, 150 were used as test sentences; the others were used as practice sentences. Of the test sentences, 90 were arbitrarily chosen to be violation sentences. In each of these sentences, 1 word was chosen to be replaced with a violation. The words were chosen from a variety of grammatical classes and sentence positions. In the sentence condition, the violations were words that, when the sentence was read at a relaxed pace, immediately appeared to be incongruous (see Table 5). For use in the both and word conditions, the incongruous words were changed to nonwords by replacing one letter. The remaining 60 test sentences were legal strings, which averaged 10.3 words in length.

The test sentences were divided into three subgroups of 50 sentences each. A subject saw all three subgroups, but each subgroup appeared in a different one of the three conditions. Thus, a subject saw all 150 test sentences, 50 in each condition, but no sentence more than once. Across sets of three subjects, all three versions of each sentence were seen.

Procedure and Apparatus. The subjects were tested individually with the apparatus used in Experiment 1 . Each condition was presented within a single block of trials. In all conditions, the subjects were instructed to read the strings as quickly as possible, and to respond "yes-problem" as soon as they encountered a problem with the string, or "no problem" as soon as they completed reading strings without problems. In all conditions, the experimenter emphasized that "yes-problem" responses should be made as soon as the problem was encountered. In the sentence condition, a problem was defined as a word that did not fit the meaning of a sentence. In the word condition, the problem was defined as a nonword (a letter string that would not form an English word). In the both condition, a problem was defined as a nonword, which would not fit the meaning of a sentence.

Each block consisteed of 20 practice trials followed by 50 randomly ordered test trials. The subjects were given two short breaks

Table 5

Examples of Stimuli in Experiment 3

\begin{tabular}{|c|c|}
\hline \multicolumn{2}{|c|}{ Violation strings ("yes-problem" response) } \\
\hline $\begin{array}{l}\text { 1.word: } \\
\text { sentence: } \\
\text { both: }\end{array}$ & $\begin{array}{l}\text { her her she sporl about to said children troubles very. } \\
\text { She said very sport to her children about her troubles. } \\
\text { She said very sporl to her children about her troubles. }\end{array}$ \\
\hline $\begin{array}{l}\text { 2.word: } \\
\text { sentence: } \\
\text { both: }\end{array}$ & $\begin{array}{l}\text { from and ate our on cuts plate we rehief salad cold knees. } \\
\text { We ate salad and cold cuts from plates relief on our knees. } \\
\text { We ate salad and cold cuts from plates rehief on our knees. }\end{array}$ \\
\hline \multicolumn{2}{|c|}{ Legal strings ("no problem" response) } \\
\hline $\begin{array}{l}\text { 1.word: } \\
\text { sentence and } \\
\text { both: }\end{array}$ & did he but not her her to wanted dare to touch soothe shoulder he. \\
\hline \multirow[t]{2}{*}{$\begin{array}{l}\text { 2.word: } \\
\text { sentence and } \\
\text { both: }\end{array}$} & $\begin{array}{l}\text { He wanted to touch her shoulder, to soothe her, but he did not dare. } \\
\text { and a he pullover wore wine-colored red slacks. }\end{array}$ \\
\hline & He wore a red pullover and wine-colored slacks. \\
\hline
\end{tabular}


Table 6

Mean Scanning Time (in Milliseconds) and Percent Errors (PEs) in Experiment 3

\begin{tabular}{lcccc}
\hline Condition & Violation Strings & PE & Legal Strings & PE \\
\hline word & 2705 & 9.4 & 3595 & 5.7 \\
sentence & 3206 & 7.6 & 3277 & 8.5 \\
both & 2343 & 4.2 & 2872 & 4.7 \\
\hline
\end{tabular}

within each block. Across subjects, all possible orders of conditions were used an equal number of times. The subjects used their dominant hands to respond "yes-problem," and error feedback was given.

Subjects. Thirty-nine subjects from the same pool as those in the previous experiments were used. Three subjects were replaced for having error rates of greater than $15 \%$, leaving a total of 36 subjects.

\section{Results and Discussion}

The main purpose of this experiment was to compare the speed of decisions in the both condition, in which word and sentence level information could be combined, with the speed in the word and sentence conditions, in which only a single level of information could be used. Analyses were first conducted on the mean scanning times for each subject. Scanning was considerably quicker in the both condition than in the other two conditions, for both violation ("yes") strings and legal ("no problem") strings. Decisions for violation strings averaged 2,343 $\mathrm{msec}$ in the both condition, which was $612 \mathrm{msec}$ faster than the mean across the word and sentence conditions $[F(1,35)=30.47, p<.001]$. Similarly, decisions for legal strings averaged $2,872 \mathrm{msec}$, which was $746 \mathrm{msec}$ faster than in the other two conditions $[F(1,35)=36.88$, $\mathrm{p}<.001]$.

The means and error rates for each condition are shown in Table 6. For scanning times, the main effect of violation condition was reliable $[\mathrm{F}(2,70)=21.39, \mathrm{p}<.001]$, as was the interaction with legality $[F(2,70)=36.72$, $p$ $<.001]$. The interaction seems to stem from the fact that, for violation strings, there were differences between conditions in the effect of violation position (to be discussed below). At this point, the important result is the strong advantage for the both condition, which held for both violation and legal strings. For error rates, there was also a main effect of condition $[\mathrm{F}(2,70)=14.08, \mathrm{p}<.001]$ and an interaction $[\mathrm{F}(2,70)=7.48, \mathrm{p}<.01]$. Because scanning was more accurate in the both condition than in the other conditions, the error data will not be discussed further.

Formal test of predictions. The main question is how much faster the both condition is than the other two conditions. In particular, we can test the interactive model's prediction that decisions in the both condition can be faster than would be expected if they were determined by separate, autonomous word and sentence level decisions. The test involves a comparison of the fastest decisions in the both condition and the fastest decisions in a distribution obtained by pooling the data from the word and sentence conditions (see Appendix or Miller, 1981, 1982, for details).

Each subject's correct scanning times were rank ordered and converted to percentiles within the both condition and within the pooled conditions. Means were taken across each percentile. The means for the lower (faster) percentiles are shown in Figure 1 for violation strings and in Figure 2 for legal strings. In Figures 1 and 2, a faster distribution appears to the left of a slower distribution. If decisions in the both condition were determined by autonomous processing at the word and sentence levels, then the fastest percentiles in the both condition should not be faster than the corresponding percentiles in the pooled data. However, if word and sentence level information is combined to mutually constrain word processing, then the percentiles in the both condition could be faster.

As can be seen in Figure 1, for violation strings, about half of the percentiles in the both condition were faster than the fastest percentiles in the word and sentence conditions, with the advantage for the both condition exceeding

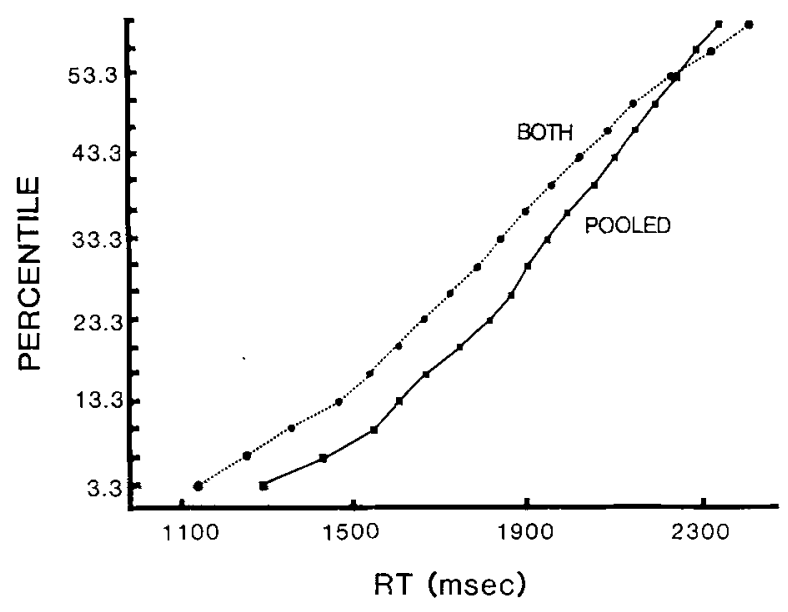

Figure 1. The cumulative probability function for violation strings.

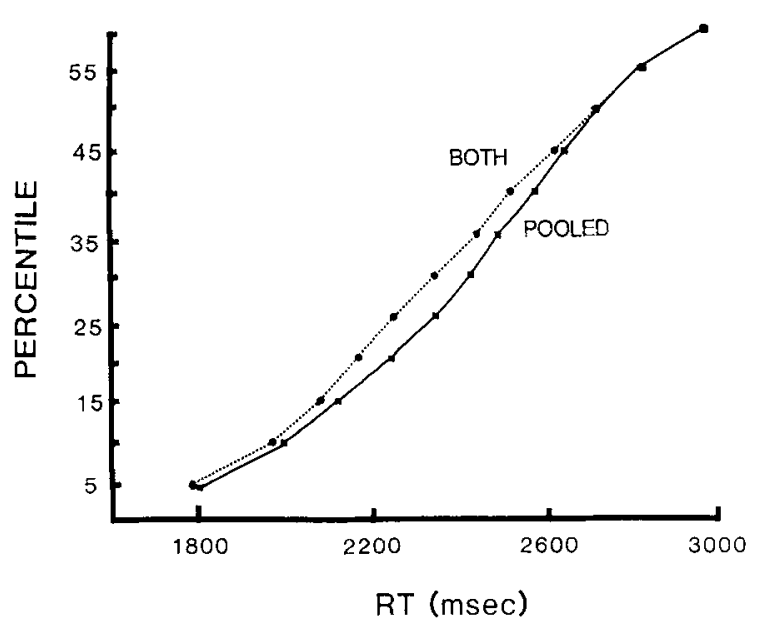

Figure 2. The cumulative probability function for legal strings. 


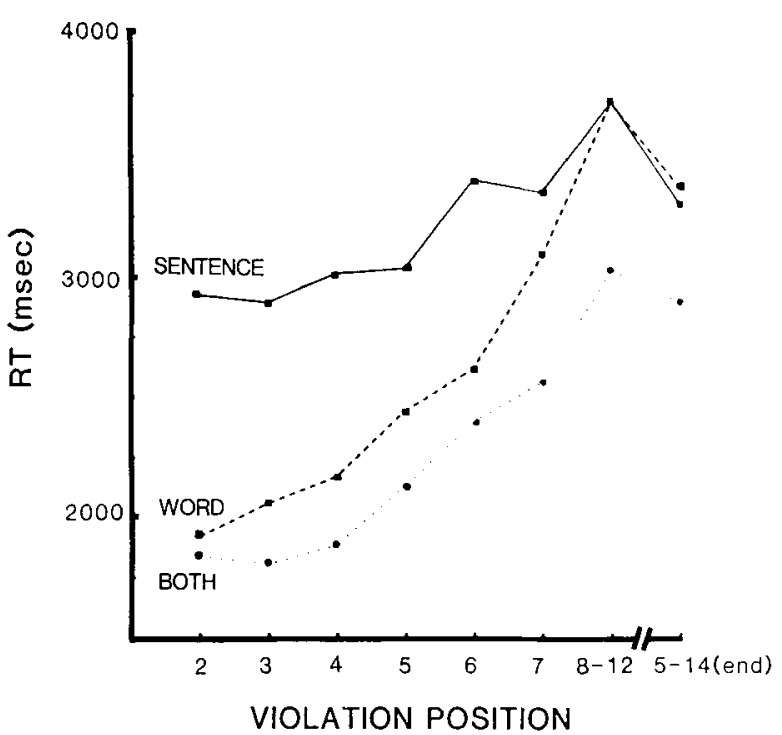

Figure 3. Scanning times for violation strings, broken down by violation position.

100 msec up through the 33 rd percentile. The differences at the first 3 percentiles were reliable $[\operatorname{ts}(35)=2.83,3.34$, and 2.96 , respectively, each $\mathrm{p}<.05$, two-tailed], but the other differences were marginally reliable or unreliable (ps >.05). In Figure 2, for legal strings, a substantial proportion of the percentiles in the both condition are faster than those in the pooled conditions. However, the differences are smaller than for the violation strings, and none were reliable ( $\mathrm{ps}>.05$ ). The consistent trends in Figures 1 and 2, together with the reliable differences, confirm the interactive model's prediction that word level and sentence level information would be combined to produce faster decisions than would be expected if the decisions were autonomous.

Analysis by violation position and string length. The relation between the position of the violation and scanning time is of particular interest because it should reveal the nature of the decision processes underlying the scanning task. If subjects scan the strings exhaustively, using a sentence level decision strategy (Forster, 1979), then scanning time should vary with the length of the string but not with the position of the violation. If the strings were scanned in a self-terminating, word-by-word manner, then scanning time should vary only with the position of the violation. The data for violation strings are presented in Figure 3, broken down by the position of the violation. Interestingly, there is a marked difference in the patterns of data. In the sentence condition, the scanning times were all rather long and increased slowly with the position of the violation. In the both and word conditions, scanning time increased steadily with the position of the violation, approaching and then, in the word condition, surpassing the scanning times in the sentence condition.
The relation between violation position, string length, and condition for the violation strings was examined further with multiple regression. In the sentence condition, length was the main predictor (predicting $27 \%$ of the variance), but position also predicted a significant proportion of the variance (a total of $37 \%$ for both factors). The weights for length and position were 94 and $83 \mathrm{msec}$, respectively. This suggests that scanning in the sentence condition was mainly exhaustive, but also somewhat selfterminating; perhaps the subjects used a mixture of these two strategies. In marked contrast, in the both and word conditions, position alone accounted for most of the variance (57\% and $69 \%$, respectively), having weights of 183 and $234 \mathrm{msec}$. Length did not predict significant additional portions of variance. This indicates that the subjects processed the strings in a self-terminating, word-byword manner in the both and word conditions. Thus, a sentence level decision strategy may be used in some situations (e.g., when decisions about word congruity are required), but it is clear that the scanning of sentences for nonwords is performed in an on-line, word-by-word manner.

The data for violation strings also attest to the robustness, across violation positions, of the advantage in the both condition. As can be seen in Figure 3, scanning was considerably faster in the both condition, at all violation positions.

For legal strings, the entire string must be scanned before a correct decision can be made. The scanning times for legal strings are shown in Figure 4, broken down by the length of the string. As can be seen, scanning time increased steadily with the length of the string, in all three of the conditions. Thus, it appears that legal strings were scanned exhaustively and that each additional word in the string added some time. Also, the advantage in the both

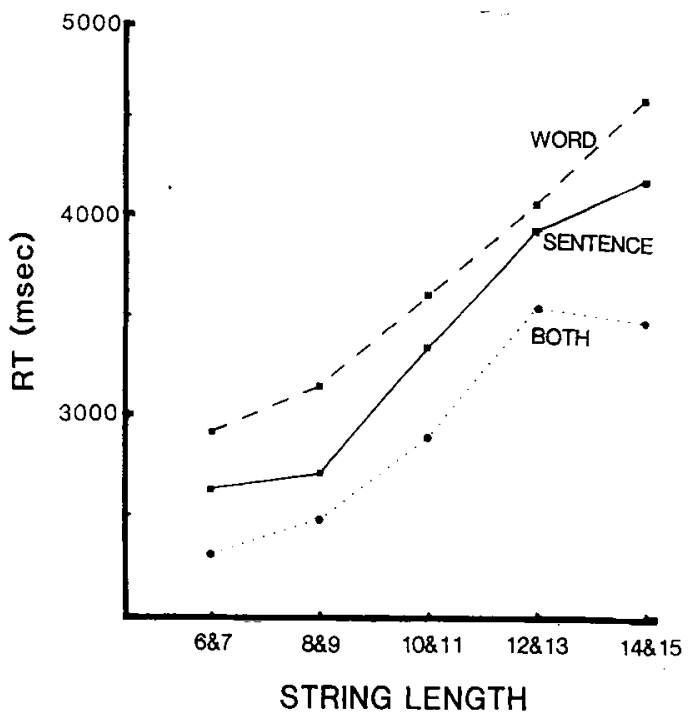

Figure 4. Scanning times for legal strings, broken down by string length. 
condition was again quite robust, holding across all string lengths.

Additional findings. For comparison with the previous experiments, we calculated the average reading speed for legal strings (in the both condition) and the size of the advantage for legal strings (in the both condition) over legal scrambled strings. The reading rate was 215 words per minute; again, this is well within the range of normal reading rates. The sentence advantage averaged $70 \mathrm{msec}$ per word. The reading rate was somewhat slower than the rate in Experiment 1 ( 235 words per minute), whereas the sentence advantage was somewhat larger ( $50 \mathrm{msec}$ per word in Experiment 1). These differences make sense in light of the finding that context effects are usually larger with more difficult words (i.e., words that are longer and of lower frequency; Stanovich \& West, 1983b). A comparison of the sentences used in Experiments 1 and 3 indicates that the more natural sentences used in Experiment 3 include some difficult words, whereas the sentences used in Experiment 1 consist mainly of easy words. Thus, with some difficult words, reading would be slower and context effects should be greater.

One interesting additional finding was that responses to legal strings were $405 \mathrm{msec}$ faster in the both condition than in the sentence condition $[F(1,35)=22.74, p<$ .001 ; see also Figure 4), even though the strings appearing in the two conditions were identical (see Table 5). Given that the stimuli did not differ between conditions, the advantage must be due to differences in the decision processes. Such a difference is in fact predicted by the interactive model, because the model assumes that word and sentence level information combine during reading to produce information that is more reliable than either type of information alone. With more reliable information, the decision criterion can be relaxed and decisions can be made sooner.

\section{GENERAL DISCUSSION}

The present experiments validated a new method for investigating the effects of contextual information on word identification. Most previous research on context effects has measured the speed of processing single words. However, as we have argued, certain types of contextual facilitation during reading may arise because adjacent words can be processed in an overlapping cascaded or parallel manner. These types of facilitation may occur only when subjects read entire phrases and sentences, for which cascaded and parallel processing is possible.

In the present experiments, we found robust facilitation effects, ranging up to $70 \mathrm{msec}$ per word, averaged across all types of words in sentences. These findings contrast with arguments, based on sentence priming studies, that the meaning of the context does not facilitate word identification (e.g., Fischler \& Bloom, 1979; Forster, 1981; Stanovich \& West, 1983a, 1983b).

The data indicate that the scanning task is natural and representative of actual reading. The subjects were able to scan quickly, at rates ( 215 to 243 words per minute) that are typical of normal reading. This quickness is important because many other findings of facilitation can be attributed to task conditions that retard the speed of word identification (see Mitchell, 1982, and Stanovich \& West, 1983b). Because scanning is fast, it is less likely that taskspecific strategies for using context (see, e.g., Forster, 1981, Mitchell, 1982, and Neely, 1977) would have time to come into play. In addition, we conclude that our subjects were reading the sentence strings for meaning, because all of the results are consistent with the assumption that word identification was constrained by both stimulus analyses and analyses of the meaning of the context.

\section{An Interactive Model}

Mutual constraints are central to the interactive model (Marslen-Wilson \& Welsh, 1978; Oden, 1984; Rumelhart \& McClelland, 1982). Experiment 2 indicates that contextual constraints include not only "narrow" semantic constraints on the exact meaning of phrases, but also, and perhaps to a greater degree, general syntactic constraints on words in phrases. Experiment 3 indicates the power of these constraints: Decisions about words were faster when word level and sentence level information could be combined than decisions that would result from a race of autonomous word level and sentence level processes.

The "interactions" within the model are subtle and yet powerful. As noted above, the word identification process involves a stimulus analysis that is independent of the context, a contextual evaluation of words in the cohort, and a process in which the results of the stimulus analysis and the contextual evaluation are combined (Marslen-Wilson \& Welsh, 1978; Oden, 1984; Rumelhart \& McClelland, 1982). These processes cascade (McClelland, 1979) to form the following time course: The initial analysis of a word causes the activation of words and their meanings, with little or no effect of context (see Oden \& Spira, 1983, and Swinney, 1979). As stimulus analyses continue and contextual analyses begin, a second phase begins that can be termed word resolution. Resolution may sometimes end gradually, being marked by an increasing probability that the dominant word candidate will continue to dominate. In this model, word identification is interactive in the sense that the resolution of a word's identity depends on both visual and contextual analyses. By using such mutual constraints, the system gains flexibility and robustness. When either stimulus or contextual information is strong, a word's identity will be resolved quickly. But when both types of information are weak, the resolution phase may be extended somewhat, until newly computed stimulus or contextual information can compensate for the initially weak information. Such temporal flexibility would be important during the processing of sequences of words (e.g., sentences) but probably would not be important during the processing of a single, isolated word.

\section{Autonomous Models of Word Identification}

The present results are inconsistent with the claim that word identification during reading is normally an autonomous process that is completed before analyses of the sen- 
tence can have a facilitative effect (e.g., Forster, 1981; Mitchell, 1982; Seidenberg et al., 1982; Stanovich \& West, 1983b). These models would have to be altered to handle the present results.

Perhaps the clearest and most influential autonomous model is described by Forster (1979; see Seidenberg et al., 1982, and Stanovich \& West, 1983b). In this model, there is no distinction between cohort activation and word resolution. The autonomous word recognition process uses stimulus information to produce a representation of a word; then sentence level processes operate on the representation. In addition, a general processor initiates responses in an experimental task. Consistent with the principle of autonomy, the outputs from each level are complete and qualitatively distinct, so the general processor can handle results from only one level at a time.

The results present two main problems for this model. First, the explanation of the advantage of sentences over scrambled words (Experiments 1 and 3 ) is attributed to a sentence level decision strategy, a hypothesis that was falsified in Experiment 3. Second, Experiment 3 indicated that subjects continually combine information from the word and sentence levels in making a decision, which is inconsistent with Forster's (1979) assumptions about the general processor.

Some theorists have argued that contextual facilitation is limited to priming within the word level (e.g., Stanovich \& West, 1983b). However, according to Stanovich and West (1983a, 1983b) and others (e.g., Seidenberg et al., 1984), word priming occurs only for highly related pairs of words. Thus, priming is not powerful enough to explain the broad facilitative effect of syntactic information in Experiment 2. And word priming cannot explain the sentence advantages in Experiments 1 and 3, because neither the experimenter-constructed sentences in Experiment 1 nor the natural sentences in Experiment 3 contain more than a few highly related pairs of words.

Given these problems for an autonomous model, the present results seem most easily accounted for by a model in which intermediate analyses from more than one level are continuously combined in making scanning decisions. Indeed, evidence from many other paradigms suggests that the integration of different levels of information is pervasive, if not obligatory (e.g., Anderson, 1981; Eriksen \& Schultz, 1979; Miller, 1981, 1982; Stanovich \& West, 1983b). In fact, Stanovich and West (1983b; West \& Stanovich, 1982) have developed a model in which different types of information can be continually combined. Stanovich and West (1983b) viewed their model as being in general agreement with Forster's (1979), but they "relaxed" several assumptions. Stanovich and West assumed that autonomous processors do not complete their analyses before making outputs, but instead make partial outputs available to higher levels and to the general processor. Stanovich and West also assumed that it is sometimes difficult, if not impossible, for the general processor to avoid combining different levels of information (West \& Stanovich, 1982).
It is not clear that the Stanovich and West model is consistent with the principle of autonomy. In particular, we question the assumption that the general processor could obtain and interpret intermediate results of the autonomous word identification process. It seems that, in order to interpret intermediate results, the general processor would have to possess some of the same knowledge as the autonomous word identifier. (At least, the processor would need more word level knowledge than if it did not interpret intermediate outputs.) Furthermore, if the word identification process is normally autonomous and its knowledge is not combined with sentence level information during the well-learned habit of reading, then it does not seem that combining information should be easy (even obligatory) during a brief experimental session. A more attractive alternative, in our view, is to assume that people are highly skilled at integrating different sources of information, and to incorporate this ability directly into the model.

In sum, the marked differences in processing times for sentences and scrambled words indicate that there is something interesting about the way words in sentences are processed-something that is not apparent when words are presented individually. The interactive model's account of the advantage for words in sentences is a hypothesis that can be tested and elaborated in further studies that allow for cascaded and parallel word processing.

\section{REFERENCES}

ANDERSON, N. H. (1981). Foundations of information integration the ory. New York: Academic Press.

Balota, D. A., \& RAYNER, K. (1983). Parafoveal visual information and semantic contextual constraints. Journal of Experimental Psychology: Human Perception and Performance, 9, 726-738.

BECKER. C. A. (1980). Semantic context effects in visual word recognition: An analysis of semantic strategies. Memory \& Cognition, 6 , 493-512.

Colins, A. M., \& Loftus, E. F. (1975). A spreading-activation the ory of semantic processing. Psychological Review, 82, 407-428.

Ehrlich, K., \& Rayner, K. (1983). Pronoun assignment and semantic integration during reading: Eye movements and immediacy of processing. Journal of Verbal Learning and Verbal Behavior, 22, 75-77.

EHRLICH, S. F., \& RAYNER, K. (1981). Contextual effects on word per ception and eye movements during reading. Joumal of Verbal Learning and Verbal Behavior, 20, 641-655.

ERIKSEN, C. W., \& SChultz, D. W. (1979). Information processing in visual search: A continuous flow conception and experimental results. Perception \& Psychophysics, 25, 249-263.

Fischler, 1., \& Bloom, P. A. (1979). Automatic and attentional processes in the effects of sentence contexts on word recognition. Journal of Verbal Learning and Verbal Behavior, 5, 1-20.

FORSTER, K. I. (1979). Levels of processing and the structure of the language processor. In W. E. Cooper \& E. Walker (Eds.), Sentence processing: Psycholinguistic studies presented to Merrill Garrett (pp. 27-85). Hillsdale, NJ: Erlbaum.

Forster, K. I. (1981). Priming and the effects of sentence and lexical contexts on naming time: Evidence for autonomous lexical processing. Quarterly Journal of Experimental Psychology, 33A, 465-495.

KIGER, J. I., \& GlASs, A. (1983). The facilitation of lexical decisions by a prime occurring after the target. Memory \& Cognition, 11 , 356-365. 
Marslen-Wilson, W. D., \& Welsh, A. (1978). Processing interactions and lexical access during word recognition in continuous speech. Cognitive Psychology, 10, 29-63.

MCClelland, J. L. (1979). On the time relations of mental processes: An examination of systems of processes in cascade. Psychological Review, 86, 287-300.

MCClELLAND, J. L., \& O'Regan, J. K. (1981). Expectation increases the benefit derived from parafoveal visual information in reading words aloud. Journal of Experimental Psychology: Human Perception and Performance, 7, 634-644.

MILLER, J. (1981). Global precedence in attention and decision. Journal of Experimental Psychology: Human Perception and Performance, 9, 1161-1174.

Miller, J. (1982). Divided attention: Evidence for coactivation with redundant signals. Cognitive Psychology, 14, 247-279.

MITCHELL, D. C. (1982). The process of reading: A cognitive analysis of fluent reading and learning to read. New York: Wiley.

Mitchell, D. C., \& Green, D. W. (1978). The effects of context and content on immediate processing in reading. Quarterly Journal of Experimental Psychology, 30, 609-636.

Mozer, M. C. (1983). Letter migration in word perception. Journal of Experimental Psychology: Human Perception and Performance, 9, 531-547.

NeELY, J. H. (1977). Semantic priming and retrieval from lexical memory: Roles for inhibitionless spreading activation and limited capacity attention. Journal of Experimental Psychology: General, 106, 226-254.

ODEN, G. C. (1983). On the use of semantic constraints in guiding syntactic analyses. International Journal of Man-Machine Studies, 19. 335-357.

ODEN, G. C. (1984). Integration of fuzzy linguistic information in language comprehension. Fuzzy Sets and Systems, 14, 29-41.

ODEN, G. C., \& SPIRA, J. L. (1983). Influence of context on the activation and selection of ambiguous word senses. Quarterly Journal of Experimental Psychology, 35A, 51-64.

RAYNER, K. (1983). Eye movements in reading. Hillsdale, NJ: Erlbaum. RueckL, J., \& OdeN, G. C. (1983, May). Integration of semantic and featural information during word identification. Presented at the meeting of the Midwestern Psychological Association, Chicago.

Rumelhart, D. E., \& MCClellaNd, J. L. (1982). An interactive activation model of context effects in letter perception: Part 2. The contextual enhancement effect and some tests and extensions of the model. Psychological Review, 89, 60-94.

SANOCKI, T., \& OdEN, G. C. (1984). Contextual validity and the effects of low constraint sentence contexts on lexical decisions. Quarterly Journal of Experimental Psychology, 36A, 145-156.

SchVaneveldT, R. W., \& McDonald, J. E. (1981). Semantic context and the encoding of words. Journal of Experimental Psychology: Human Perception and Performance, 7, 673-687.

Schwanenflugel, P. J., \& Shoben, E. J. (in press). The influence of sentence constraint on the scope of facilitation for upcoming words. Journal of Verbal Learning and Verbal Behavior.

SeidenberG, M. S., Tanenhaus, M. K., Leiman, J. L., \& BIENKowSKI, M. (1982). Automatic access of the meanings of ambiguous words in context: Some limitations of knowledge-based processing. Cognitive Psychology, 14, 489-537.

SeidenberG, M. S., Water, G. S., SANders, M., \& Langer, P. (1984). Pre- and postlexical loci of contextual effects on word recognition. Memory \& Cognition, 12, 315-328.

SмIтн, F. (1971). Understanding reading: A psycholinguistic analysis of reading and learning to read. New York: Holt, Rinehart \& Winston.

STANOVICH, K. E., \& WEST, R. F. (1983a). The generalizability of context effects on word recognition: A reconsideration of the roles of parafoveal information and sentence context. Memory \& Cognition. $11,49-58$.

Stanovich, K. E., \& WeSt, R. F. (1983b). On priming by a sentence context. Journal of Experimental Psychology: General, 112, 1-36.

SWINNEY, D. A. (1979). Lexical access during sentence comprehension: (Re)consideration of context effects. Journal of Verbal Learning and Verbal Behavior, 18, 645-661.
Tulving, E., \& Gold, C. (1963). Stimulus information and contextual information as determinants of tachistoscopic recognition of words. Journal of Experimental Psychology, 66, 319-327.

UNDERWOOD, G., \& BARGH, K. (1982). Word shape, orthographic regularity, and contextual interactions in a reading task. Cognition, 12, 197-209.

West, R. F., \& STaNovich, K. E. (1982). Source of inhibition in experiments on the effect of sentence context on word recognition. Journal of Experimental Psychology: Learning, Memory, and Cognition, 8, 385-399.

Zol.A, D. (1984). Redundancy and word perception during reading. Perception \& Psychophysics, 36, 277-284.

\section{NOTES}

1. Our a priori predictions concern this specific comparison. In general, the other data (error rates and data for distractor strings) are of interest only insofar as they suggest conclusions contrary to those indicated by the main comparison. The possible effects of legality (response) were not tested because a difference would be hard to interpret; any such effect could be an artifact, due to the unequal response probabilities.

2 . To check the consistency of effects across items, informal tests were conducted by repeating pairwise comparisons with items treated as if it were a random factor. All differences reported herein were consistent across items.

3. Fourteen pilot subjects determined which context most strongly predicted the meaning of the target and then rated how much more strongly, on a scale from 1 ("about the same") to 7 ("very much more strongly"). All subjects agreed that all of the expectancy contexts were more predictive than the plausible contexts; the mean rating was 4.9 .

4. Several considerations make it unlikely that the semantic effect can be caused by a possible confound, namely, properties of the contexts, other than degree of predictiveness, that differ between the contexts. Because many pairs of predictive and plausible contexts differed in grammatical structure, it is possible that the effect could be due to differences in grammatical structure. This possibility was evaluated by comparing scanning times for a subset of 13 sentence sets for which the contexts had similar surface structures (e.g., see the last two examples in Table 3). Facilitation was also obtained with this subset; in fact, the effect $(187 \mathrm{msec})$ was larger with these contexts than with the other contexts. The contexts may have differed on other properties as well, such as word difficulty or number of words. However, the contexts were constructed to be generally similar in difficulty and length. Moreover, the subjects could read the contexts at their own pace, and there was an interval of about $1 \mathrm{sec}$ between the offset of the context and the onset of the target phrase. Thus, any differences between the contexts probably would have been "absorbed" before the target appeared.

\section{APPENDIX Description of Formal Test}

The rationale for the test turns on an inequality that follows from the autonomous model. Consider the probabilities that, before some time $\mathrm{T}$, an autonomous word level process would complete its decision, $\mathrm{p}(\mathrm{W}<\mathrm{T})$, an autonomous sentence level process would complete its decision, $\mathrm{p}(\mathrm{S}<\mathrm{T})$, and either level would complete its decision, $\mathrm{p}[(\mathrm{W}<\mathrm{T})$ or $(\mathrm{S}<\mathrm{T})]$. The probabilities are related by an elementary law of probability,

$\mathrm{p}[(\mathrm{W}<\mathrm{T})$ or $(\mathrm{S}<\mathrm{T})]=\mathrm{p}(\mathrm{W}<\mathrm{T})+\mathrm{p}(\mathrm{S}<\mathrm{T})-[\mathrm{p}(\mathrm{W}<\mathrm{T}) \cdot \mathrm{p}(\mathrm{S}<\mathrm{T})]$.

Since the rightmost term of the equation could never be less than zero, we can delete it and form the inequality,

$$
\mathrm{p}[(\mathrm{W}<\mathrm{T}) \text { or }(\mathrm{S}<\mathrm{T})] \leq \mathrm{p}(\mathrm{W}<\mathrm{T})+\mathrm{p}(\mathrm{S}<\mathrm{T}) \text {. }
$$


The inequality states that, for a given time $T$, the probability that a subject made a decision in the both condition (i.e., either the word level or the sentence level completed its decision) can never be greater than the sum of (1) the probability that the subject decided in the word condition and (2) the probability that the subject decided in the sentence condition. The autonomous model predicts that this inequality will hold. In contrast, in the interactive model, word level and sentence level information are combined so that the two types of information in combination can sometimes produce a decision before a decision can be made at either level alone. Therefore, the probability of a decision in the both condition at time $\mathrm{T}$ can exceed the sum of the other two probabilities.

The prediction can be tested by comparing the fastest part of the cumulative probability function for the both condition against the fastest part of the cumulative function obtained by pooling the data from the word and sentence conditions. The analysis holds for only the fastest parts of the data because, as $\mathrm{T}$ becomes large, the sum of the probabilities of responding in either the word or the sentence condition approaches 2 , whereas the prob- ability of responding in the both condition approaches only 1 . Therefore, it is increasingly unlikely that the inequality could be violated as $\mathrm{T}$ becomes large.

Scanning times for correct responses were used in the analysis. The times were pooled across the word and sentence conditions, for each subject. Then each subject's times were rank ordered within the pooled condition and within the both condition, for each response. The rank orders correspond to percentiles, ranging up to near the 100th percentile in the both condition and near the 200th percentile in the pooled conditions. (The top end of the range varies because the data for error trials were omitted from the analysis.) These rank ordered observations correspond to probabilities of responding before time $\mathrm{T}$. For example, if a subject's $30 \mathrm{th}$ percentile was $2,200 \mathrm{msec}$, then the probability of responding by $\mathrm{T}=2,200$ would be .30 .

(Manuscript received September 20, 1984; revision accepted for publication March 4, 1985.) 